\title{
Differential expression of immunohistochemical markers in bladder smooth muscle and myofibroblasts, and the potential utility of desmin, smoothelin, and vimentin in staging of bladder carcinoma
}

\author{
Leona Council ${ }^{1}$ and Omar Hameed H,2,3 $^{1,2}$
}

\author{
${ }^{1}$ Division of Anatomic Pathology, Department of Pathology, University of Alabama at Birmingham, \\ Birmingham, $A L, U S A ;{ }^{2}$ Department of Surgery, University of Alabama at Birmingham, Birmingham, $A L$, \\ USA and ${ }^{3}$ Comprehensive Cancer Center, University of Alabama at Birmingham, Birmingham, AL, USA
}

\begin{abstract}
Distinguishing bladder muscularis propria from muscularis mucosae can be problematic especially in transurethral resection specimens performed for bladder carcinoma. Moreover, bladder carcinoma can be associated with a proliferative/desmoplastic myofibroblastic response that can resemble smooth muscle and potentially lead to overdiagnosis of muscularis propria invasion. The aim of this study was to investigate the potential role of immunohistochemistry in staging bladder carcinoma by evaluating the expression of different markers in myofibroblasts and nonvascular smooth muscle cells in 15 cases of invasive bladder carcinoma. Reactive myofibroblasts were consistently positive for vimentin and smooth muscle actin, consistently negative for caldesmon, desmin, and smoothelin, and had variable expression of actin and CD10. Nonvascular smooth muscle cells of the bladder were consistently positive for smooth muscle actin, actin, desmin, and caldesmon, and consistently negative for CD10. In contrast to smooth muscle cells of the muscularis propria, which displayed strong smoothelin expression in all 15 cases, the smooth muscle cells of the muscularis mucosae displayed moderate smoothelin expression in only $1(9 \%)$ of 11 cases $\left(P=10^{-7}\right)$. Surprisingly, although strongly highlighting endothelial and endomysial cells, the smooth muscle cells of the muscularis propria weakly expressed vimentin in only $1(7 \%)$ of 15 cases, whereas smooth muscle cells of the muscularis mucosae had moderate or strong expression in $9(82 \%)$ of 11 cases $(P=0.00016)$. The sensitivity and specificity of desmin or caldesmon expression for smooth muscle cells were $100 \%$. The sensitivity and specificity of strong smoothelin expression for muscularis propria were $100 \%$, whereas those of absent vimentin expression were 93 and $82 \%$, respectively. Although morphology remains the gold standard, the findings suggest that immunohistochemistry, using a panel composed of desmin, smoothelin, and vimentin, may be potentially useful for staging of bladder carcinoma. Confirmatory larger-scale studies, especially on transurethral resection specimens, are warranted.
\end{abstract}

Modern Pathology (2009) 22, 639-650; doi:10.1038/modpathol.2009.9; published online 27 February 2009

Keywords: bladder carcinoma; staging; immunohistochemistry; smoothelin; desmin; vimentin

Carcinoma of the urinary bladder is a significant cause of morbidity and mortality in the United

Correspondence: Dr Omar Hameed, MBChB, Division of Anatomic Pathology, Department of Pathology, University of Alabama at Birmingham, 619 19th St South, NP 3550, Birmingham, AL 35294-6823, USA.

E-mail: ohameed@uab.edu

Presented in part at the 97th Annual Meeting of the United States and Canadian Academy of Pathology, Denver, CO, USA, March 2008.

Received 25 August 2008; revised and accepted 6 February 2009; published online 27 February 2009
States where it is likely that more than 51000 new cases were diagnosed in 2008. Of these, approximately $20 \%$ are estimated to be fatal. ${ }^{1}$ The single most important prognostic factor in urothelial carcinoma is pathological stage, ${ }^{2}$ which includes the anatomic depth of invasion on which major therapeutic decisions are made. Similar to noninvasive tumors, a carcinoma confined to the lamina propria (LP) is usually treated conservatively, whereas one extending into the muscularis propria almost always dictates more radical surgical management. Accordingly, identification of invasion, 
and determining its depth if present, is an essential component in the evaluation of bladder biopsies and transurethral resection of bladder tumor specimens that harbor carcinoma. This, however, is not always straightforward and both understaging and overstaging have been reported to occur in a significant proportion of cases. ${ }^{3-5}$ Although the usually thin, wispy, and often discontinuous smooth muscle fibers of the muscularis mucosae that are closely associated with the blood vessels of the LP can readily be distinguished from the thick smooth muscle bundles of the muscularis propria in cystectomy specimens and well-oriented bladder biopsies, ${ }^{6-9}$ this is not always the case in transurethral resection specimens. The fragmentation, tangential sectioning, and thermal artifacts inherent to these specimens, as well as the inflammatory and desmoplastic responses often elicited by invasive tumors, can lead to poor orientation and difficulty in distinguishing the type of smooth muscle invaded by carcinoma (muscularis mucosae vs muscularis propria) and accurately determining the depth of invasion. ${ }^{10,11}$ Moreover, recent studies ${ }^{7,8}$ have emphasized the fact that the muscularis mucosae, not infrequently, can have a thickened hyperplastic appearance that may resemble the thick bundles of the muscularis propria, especially in transurethral resection specimens and may also cause difficulty in staging of cancers in these specimens.

Another potential problem in staging bladder carcinomas is that, similar to invasive carcinomas elsewhere, these tumors are often associated with a variably cellular, spindle-cell myofibroblastic stromal response. This is usually desmoplastic in nature, but more cellular patterns including a pseudosarcomatous stromal response may also be seen. ${ }^{11,12}$ For the most part, these myofibroblasts can be readily distinguished from the smooth muscle cells of the muscularis mucosae or muscularis propria; however, we have seen cases of bladder carcinoma misdiagnosed as muscularis propriainvasive disease based solely on the presence a cellular myofibroblastic response surrounding the tumor, including one where immunoreactivity for smooth muscle actin was used to 'support' the diagnosis of muscularis propria invasion. This raises several important questions: can immunohis- tochemistry help distinguish between reactive myofibroblasts and smooth muscle cells of the bladder, or, given the importance of the issue, between muscularis mucosae and muscularis propria; and, if so, can immunohistochemistry be used as an adjunct in staging carcinomas of the bladder, especially in the circumstances discussed above?

Although the use of immunohistochemistry in the evaluation of spindle-cell tumors and tumor-like proliferations of the bladder is well established, ${ }^{13}$ to the best of our knowledge its potential function, if any, in staging bladder carcinoma has not been fully evaluated. To address this issue, the immunohistochemical expression of different potentially discriminatory markers in myofibroblasts and smooth muscle cells of the muscularis mucosae and muscularis propria from cystectomy specimens was evaluated and presented herein.

\section{Materials and methods}

After approval by the University of Alabama at Birmingham Institutional Review Board, 15 consecutive cystectomy specimens resected at the University Hospital for invasive bladder carcinoma were reviewed to identify representative sections of muscularis propria-invasive tumor associated with a myofibroblastic response and/or muscularis mucosae adjacent to or involved by tumor. We purposefully limited this study to cystectomy specimens as, in contrast to some transurethral resection specimens, the depth of invasion and type of muscle can readily be determined based on hematoxylin and eosin-stained sections. As is the case in routine practice, this morphologically determined depth of invasion/type of muscle was considered to be the gold standard.

Immunohistochemistry was then performed on sections from the corresponding paraffin blocks using an automated immunostainer (Benchmark XT; Ventana Medical Systems, Tuscon, AZ, USA or Autostainer 720; Lab Vision Corporation, Freemont, CA, USA). The antibodies used, their clones, dilutions, and sources are presented in Table 1. Most of these antibodies were chosen because (1) they are known to be expressed in mesenchymal

Table 1 Immunohistochemical stains used in the study with their clones, dilutions and manufacturers ${ }^{\mathrm{a}}$

\begin{tabular}{llll}
\hline Antibody & Clone & Dilution & Manufacturer \\
\hline SMA & 1A4 & Prediluted & Cell Marque, Rocklin, CA, USA \\
Actin & HUC1-1 & Prediluted & Ventana Medical Systems \\
Caldesmon & h-CD & $1: 50$ & Dako North America, Carpinteria, CA, USA \\
Desmin & DE-R-11 & Prediluted & Ventana Medical Systems \\
Smoothelin & R4A & $1: 400$ & Chemicon International, Temecula, CA, USA \\
Vimentin & 3B4 & Prediluted & Ventana Medical Systems, Tuscon, AZ, USA \\
CD10 & 56C6 & Prediluted & Ventana Medical Systems
\end{tabular}

SMA, smooth muscle actin.

${ }^{\mathrm{a}}$ Heat-induced epitope retrieval was performed on all cases using a citrate buffer for either 20 min (smoothelin) or 60 min (remainder). 
cells (vimentin), contractile cells (smooth muscle actin, actin, desmin, and caldesmon), or reactive stromal cells (CD10) in carcinomas of the blad$\operatorname{der}^{14,15}$ and elsewhere; ${ }^{16,17}$ (2) they have been previously validated in our lab and in routine use; and (3) preliminary testing (in two cases) suggested that they have the best discriminatory power among a larger group of antibodies we tested that also included cytokeratin CAM5.2, calponin, collagen type IV, and anaplastic lymphoma kinase-1 (data not shown). On the other hand, smoothelin, a novel $59 \mathrm{kDa}$ cytoskeletal protein marker that has been found to be selectively expressed in smooth muscle cells of various organs ${ }^{18}$ including the bladder, ${ }^{19,20}$ was selected after reading an abstract by Lapetino et $a l^{21}$ that suggested that it has some utility in distinguishing between muscularis mucosae and muscularis propria of the bladder.

Interpretation of immunohistochemical stains in each case was performed by semiquantitatively analyzing the intensity of staining separately in each compartment (myofibroblasts, smooth muscle of the muscularis mucosae, and smooth muscle of muscularis propria). Vascular smooth muscle was used as an internal control for all antibodies except CD10, with absent staining categorized as negative (0), and an intensity of staining that was weaker, equal to, or stronger than the intensity of staining in vascular smooth muscle categorized as weak $(1+)$, moderate $(2+)$, or strong $(3+)$ staining.

The patterns of staining in the different compartments were compared to each other and the utility of the different antibodies in distinguishing between myofibroblasts and smooth muscle cells, and between the smooth muscle of the muscularis mucosae and that of the muscularis propria was evaluated by comparing the sensitivity (true positives/true positives + false negatives), specificity (true negatives/ true negatives + false positives), and accuracy (true positives + true negatives/true positives + false positives + true negatives + false negatives) rates of each immunostain in identifying the specific components. The $95 \%$ confidence intervals (CI) for proportions based on a binomial probability distribution were also calculated to assess the accuracy of the estimates of the sensitivity, specificity, and accuracy rates of the different immunostains in labeling the compartment in question.

Statistical analysis was used to compare the categorical data obtained using either the $\chi^{2}$-test, or the Fisher's exact test, as statistically appropriate. A $P$-value of 0.05 was considered statistically significant.

\section{Results}

\section{Light Microscopic Findings}

There were 14 high-grade urothelial carcinomas and 1 squamous-cell carcinoma. Of all cases, 14 showed a variably cellular desmoplastic/myofibroblastic tumor response (including 1 with a pseudosarcomatous reaction), and 11 had residual muscularis mucosae adjacent to and/or involved by tumor.

\section{Immunohistochemical Findings (Table 2)}

Reactive myofibroblasts displayed strong vimentin expression and moderate to strong smooth muscle actin expression in all cases, with no expression of caldesmon, desmin, and smoothelin in any case (Figure 1). In addition, weak actin expression was

Table 2 Expression of different immunohistochemical markers within myofibroblastic cells, smooth muscle cells of the muscularis propria, and smooth muscle cells of the muscularis mucosae in 15 cystectomy specimens with invasive carcinoma

\begin{tabular}{|c|c|c|c|c|c|c|c|c|}
\hline & \multirow{2}{*}{$\begin{array}{l}\text { Staining } \\
\text { intensity }\end{array}$} & \multicolumn{7}{|c|}{ Immunohistochemical marker } \\
\hline & & $S M A$ & Actin & $C A L$ & $D E S$ & $S M O$ & $V I M$ & CD10 \\
\hline \multirow[t]{4}{*}{ MF cells $(n=15)$} & 0 & $0(0)$ & $7(47)$ & $15(100)$ & $15(100)$ & $15(100)$ & $0(0)$ & $4(27)$ \\
\hline & $1+$ & $0(0)$ & $8(53)$ & $0(0)$ & $0(0)$ & $0(0)$ & $0(0)$ & $3(20)$ \\
\hline & $2+$ & $0(0)$ & $0(0)$ & $0(0)$ & $0(0)$ & $0(0)$ & $0(0)$ & 2 (13) \\
\hline & $3+$ & $15(100)$ & $0(0)$ & $0(0)$ & $0(0)$ & $0(0)$ & $15(100)$ & $6(40)$ \\
\hline \multirow[t]{4}{*}{ SM cells of MP $(n=15)$} & 0 & $0(0)$ & $0(0)$ & $0(0)$ & $0(0)$ & $0(0)$ & $14(93)$ & $15(100)$ \\
\hline & $1+$ & $0(0)$ & $0(0)$ & $1(7)$ & $0(0)$ & $0(0)$ & $1(7)$ & $0(0)$ \\
\hline & $2+$ & $0(0)$ & $0(0)$ & $3(20)$ & $0(0)$ & $0(0)$ & $0(0)$ & $0(0)$ \\
\hline & $3+$ & $15(100)$ & $15(100)$ & $11(73)$ & $15(100)$ & $15(100)$ & $0(0)$ & $0(0)$ \\
\hline \multirow[t]{4}{*}{ SM cells of MM $(n=11)$} & 0 & $0(0)$ & $0(0)$ & $0(0)$ & $0(0)$ & $4(36)$ & $2(18)$ & $11(100)$ \\
\hline & $1+$ & $0(0)$ & $0(0)$ & $2(18)$ & $0(0)$ & $6(55)$ & $8(73)$ & $0(0)$ \\
\hline & $2+$ & $0(0)$ & $0(0)$ & $2(18)$ & $0(0)$ & $1(9)$ & $1(9)$ & $0(0)$ \\
\hline & $3+$ & $11(100)$ & $11(100)$ & $7(64)$ & $11(100)$ & $0(0)$ & $0(0)$ & $0(0)$ \\
\hline
\end{tabular}

SMA, smooth muscle actin; CAL, caldesmon; DES, desmin; SMO, smoothelin; VIM, vimentin; MF, myofibroblastic; SM, smooth muscle; MP, muscularis propria; MM, muscularis mucosae.

${ }^{\mathrm{a}}$ Expressed as number of cases positive and percentages (between parentheses). 

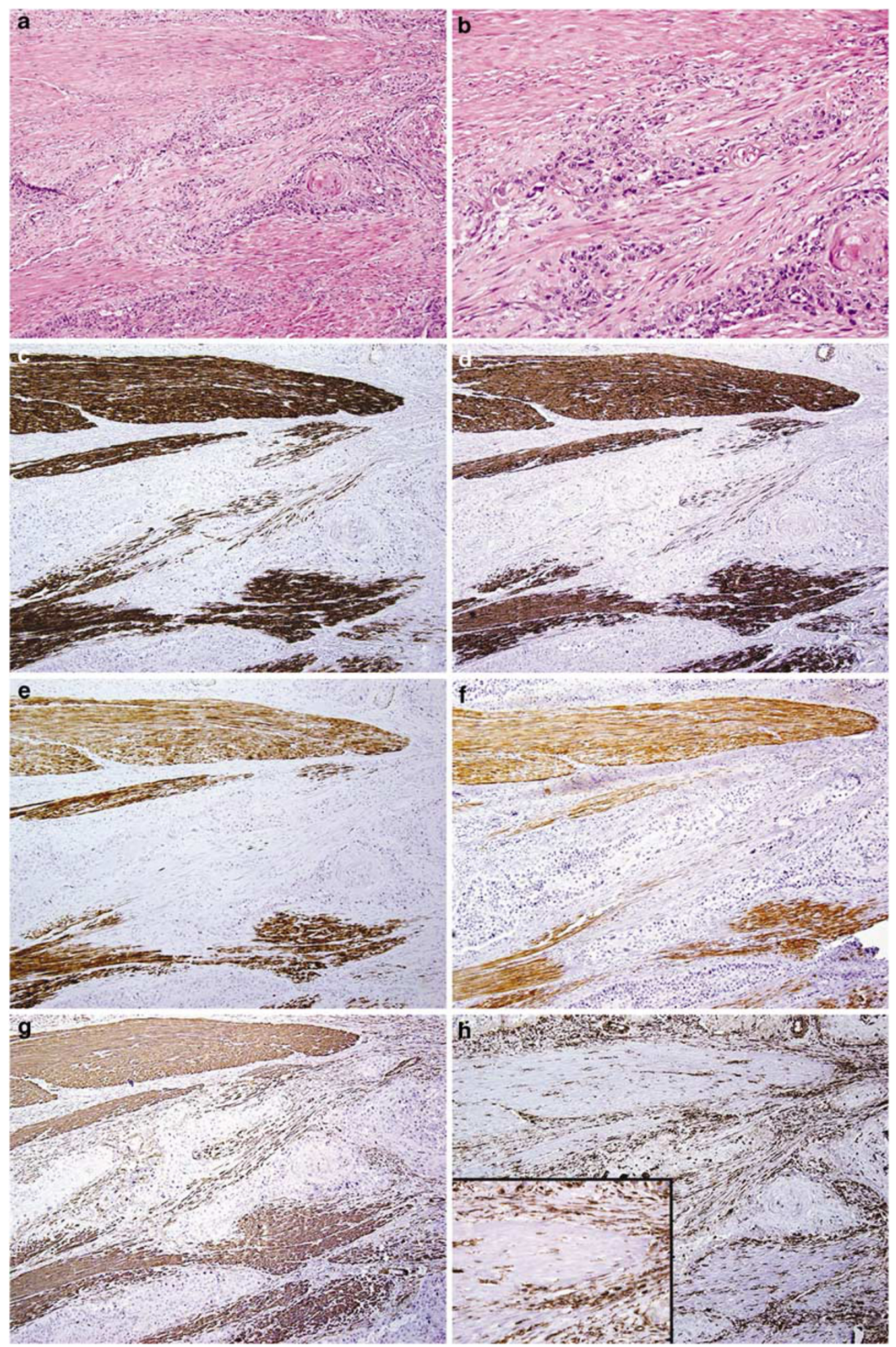

Figure 1 Expression of different immunostains in the smooth muscle of the muscularis propria and reactive myofibroblastic cells associated with invasive carcinoma. A case of squamous-cell carcinoma that is seen to invade the muscularis propria (a, b). A desmoplastic reaction is seen surrounding the tumor nests, which is composed of paler myofibroblasts with more tapered nuclei (b). Desmin (c), actin (d), caldesmon (e), and smoothelin (f) immunostains all appear to specifically highlight the smooth muscle of the muscularis propria, albeit with variable intensity, but not the myofibroblasts. On the other hand, a smooth muscle actin immunostain also labeled the myofibroblasts and endothelial cells around the tumor (g), as did a vimentin immunostain (h). Although vimentin highlighted some scattered endomysial cells within the muscle fibers, the smooth muscle cells themselves appeared negative (h, inset). A CD10 immunostain (not shown) weakly highlighted some of the myofibroblasts present. 
seen in $8(53 \%)$ cases, whereas CD10 expression, of variable intensity, was seen in $11(73 \%)$ cases.

Smooth muscle cells of the muscularis mucosae and muscularis propria had similar expression profiles for most markers; there was strong expression of smooth muscle actin, actin, and desmin, and variably intense expression of caldesmon in all cases, with no CD10 expression in any case (Figures 1-3). The expression of smoothelin and vimentin in the smooth muscle of the muscularis mucosae, however, was different from that in the smooth muscle of the muscularis propria. Smooth muscle cells of the muscularis mucosae were mostly negative (Figure 2g) or weakly expressed smoothelin; only one case ( $9 \%$ ) had moderate expression (Figure 3d). In contrast, the smooth muscle cells of the muscularis propria were strongly smoothelin positive (Figure 1f) in all 15 cases $(P=0.0000001)$. In a similar fashion, smooth muscle cells of the muscularis mucosae were positive for vimentin (Figures $2 \mathrm{~h}$ and 3c), in most cases (9 of $11 ; 82 \%$ ), whereas smooth muscle cells of the muscularis propria rarely expressed vimentin (1 of $15 ; 7 \%$ ) $(P=0.00016)$. This absent/weak vimentin expression in the muscularis propria was limited to smooth muscle cells only as moderate to strong expression was present in the endomysial and endothelial cells interspersed between the smooth muscle fibers (Figure 1h).

\section{Immunohistochemical Stains in Distinguishing Smooth Muscle Cells from Reactive Myofibroblasts (Table 3)}

When any intensity of immunoreactivity was considered positive, smooth muscle actin, actin, caldesmon, and desmin immunostains were equally effective in highlighting smooth muscle cells of both compartments (muscularis mucosae or muscularis propria), each with a sensitivity of $100 \%$. The sensitivity of smoothelin in this regard was $85 \%$, but was $100 \%$ when only smooth muscle cells of muscularis propria were considered (see below). Caldesmon, desmin, and smoothelin immunostains were more specific than smooth muscle actin and actin in highlighting smooth muscle cells, as the specificity of each of the former immunostains was $100 \%$, whereas that of smooth muscle actin was $0 \%$ $(P<0.0001)$, and that of actin was $47 \%(P=0.0003)$. Thus among the different contractile markers, caldesmon and desmin appeared to be equally effective in distinguishing between nonvascular smooth muscle cells in the bladder and reactive myofibroblasts. As noted before however, desmin, in contrast to caldesmon, consistently produced strong staining of smooth muscle cells (Table 1) and, as such, might be the better choice.

Although negative vimentin or CD10 immunostains also favored smooth muscle cells, these immunostains were less accurate than caldesmon and desmin and did not add additional discriminatory power. The utility of different immunostains in distinguishing between smooth muscle cells and reactive myofibroblasts is further illustrated in Figures 4 and 5.

\section{Immunohistochemical Stains in Distinguishing Smooth Muscle Cells of the Muscularis Propria from those of the Muscularis Mucosae (Table 4)}

Although the expression of smooth muscle actin, actin, caldesmon, and desmin in the smooth muscle cells of the muscularis propria and in those of the muscularis mucosae was identical, differential expression of smoothelin (Figure 4h) and vimentin appeared to be useful in distinguishing between the two. The sensitivity of smoothelin for labeling smooth muscle cells of the muscularis propria was $100 \%$ and its specificity was $36 \%$ when any intensity of immunoreactivity was considered positive; however, the specificity progressively increased with more intense staining cutoffs, becoming $100 \%$ when the cutoff was strong staining (Table 4). Given that vimentin was only rarely expressed in the smooth muscle cells of the muscularis propria, the sensitivity of negative or weak vimentin expression for labeling such cells was $100 \%$ but the specificity was only $9 \%$; using a negative immunoreaction as the cutoff increased the specificity to $82 \%$ whereas the sensitivity decreased to $93 \%$.

Using the two immunostains in combination also improved detection of smooth muscle cells of the muscularis propria as the sensitivity of moderate or strong smoothelin expression combined with negative vimentin expression was $93 \%$ and the specificity was $100 \%$, whereas the sensitivity of moderate or strong smoothelin expression combined with negative or weak vimentin expression was $100 \%$ and the specificity was $100 \%$.

\section{Discussion}

In this study we have shown that differential expression of immunohistochemical markers appears potentially useful in distinguishing between myofibroblasts and smooth muscle cells of the bladder, and also between the smooth muscle cells of the muscularis mucosae and those of the muscularis propria. Specifically, desmin or caldesmon appeared to be accurate immunostains that can help distinguish smooth muscle cells from myofibroblasts, whereas smoothelin and vimentin, especially if used in combination, may also help distinguish muscularis mucosae from muscularis propria. Evaluation of additional cases and confirmatory studies on transurethral resection specimens are warranted to further explore this, as well as the overall potential utility of immunohistochemistry as 



Figure 2 Expression of different immunostains in the smooth muscle of the muscularis mucosa. The smooth muscle fibers of the muscularis mucosae in this bladder (a, b) were strongly immunoreactive for desmin (c), actin (d), and smooth muscle actin (e), weakly immunoreactive for caldesmon (f), and negative for smoothelin (g). The smooth muscle fibers of the muscularis mucosae were also weakly positive for vimentin (h, inset) and negative for CD10 (not shown). 

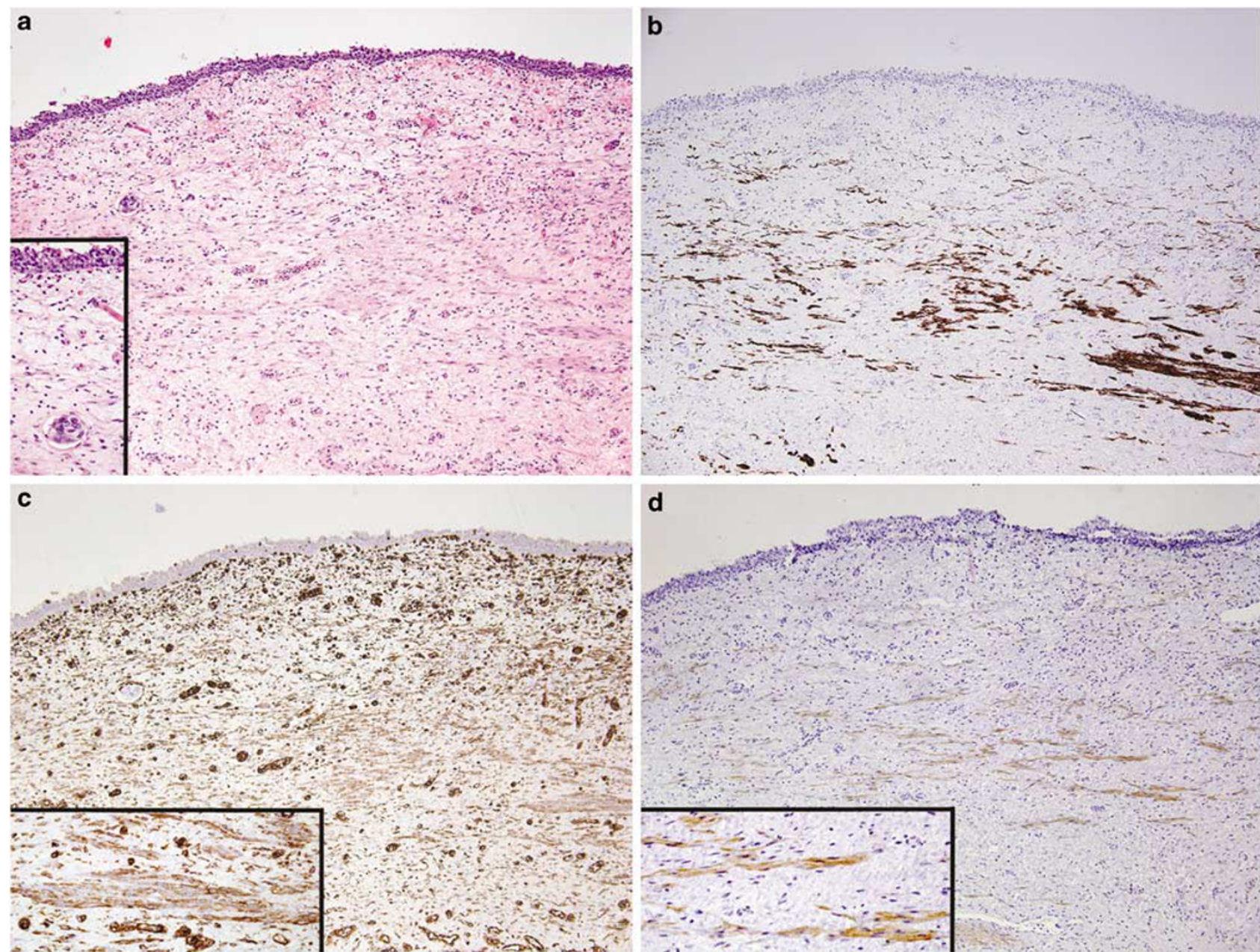

Figure 3 Smoothelin expression in the smooth muscle of the muscularis mucosae. Section of bladder showing an edematous lamina propria involved by high-grade urothelial carcinoma with angiolymphatic invasion (a, inset). In addition to being immunoreactive for desmin (b) and vimentin (c, inset), the smooth muscle fibers of the muscularis mucosae in this case displayed moderate immunoreactivity for smoothelin (d), more reminiscent of the intensity of staining associated with muscularis propria. The muscularis mucosae were also positive for other muscle markers and negative for CD10 (not shown).

Table 3 The sensitivity, specificity, and accuracy rates of different immunohistochemical markers in distinguishing nonvascular smooth muscle cells of the bladder from reactive myofibroblasts associated with invasive carcinoma

\begin{tabular}{|c|c|c|c|c|}
\hline Marker & Result cutoff & $\begin{array}{l}\text { Sensitivity (\%) } \\
(95 \% \text { CI) }\end{array}$ & $\begin{array}{c}\text { Specificity (\%) } \\
(95 \% \text { CI })\end{array}$ & $\begin{array}{c}\text { Accuracy }(\%) \\
(95 \% \text { CI })\end{array}$ \\
\hline SMA & Positive (any intensity) & $100(84-100)$ & $0(0-25)$ & $63(47-77)$ \\
\hline Actin & Positive (any intensity) & $100(84-100)$ & $47(22-73)$ & $80(65-91)$ \\
\hline Caldesmon & Positive (any intensity) & $100(84-100)$ & $100(75-100)$ & $100(91-100)$ \\
\hline Desmin & Positive (any intensity) & $100(84-100)$ & $100(75-100)$ & $100(91-100)$ \\
\hline Smoothelin & Positive (any intensity) & $85^{\mathrm{a}}(66-94)$ & $100(75-100)$ & $90^{\mathrm{a}}(76-97)$ \\
\hline Vimentin & Negative & $62(41-79)$ & $100(75-100)$ & $76(59-87)$ \\
\hline CD10 & Negative & $100(84-100)$ & $73(45-91)$ & $90(76-97)$ \\
\hline
\end{tabular}

CI, confidence interval; SMA, smooth muscle actin.

${ }^{\mathrm{a}}$ Both the sensitivity and accuracy of smoothelin increased to $100 \%$ when only smooth muscle of the muscularis propria was considered.

a potential aid to morphology in staging of bladder carcinoma.

The evaluation of bladder lesions usually entails obtaining cold biopsy and transurethral resection specimens for histopathological examination. One of the requirements for adequate evaluation of these specimens is the determination of extent of involvement or pathological stage when carcinoma is present. ${ }^{22}$ Such carcinoma may be limited to the epithelium (noninvasive papillary carcinoma (pTa) or carcinoma in situ (pTis)), extend into the subepithelial connective tissue/LP (pT1), invade 

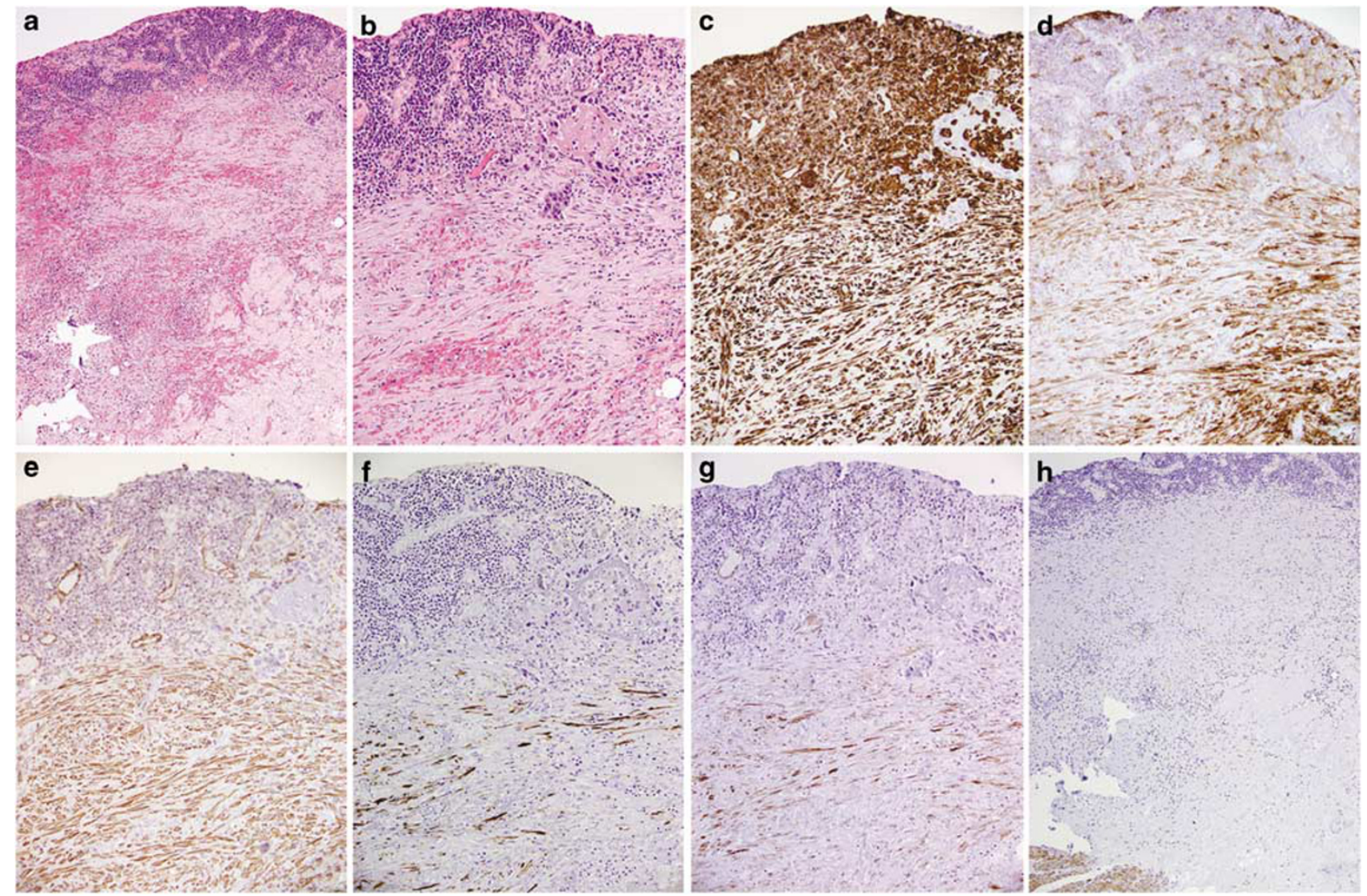

Figure 4 The utility of different immunostains in distinguishing the smooth muscle of the muscularis mucosae from reactive myofibroblasts and the utility of smoothelin in specifically highlighting the muscularis propria. A section of bladder showing high-grade urothelial carcinoma within the lamina propria associated with a dense lymphoplasmacytic infiltrate and a spindle-cell fibroblastic reaction $(\mathbf{a}, \mathbf{b})$ that renders identification of individual smooth muscle fibers of the muscularis mucosae very difficult. The diffuse patterns of immunoreactivity within the spindle-cell area for vimentin (c), CD10 (d), and smooth muscle actin (e) in this setting do not appear to be of much help in distinguishing smooth muscle cells from myofibroblasts. On the other hand, the patterns of staining obtained with desmin (f) and less so for actin (g) seem to be more helpful in this regard as they appear to highlight two populations of spindle cells: dispersed individual or clustered positive cells presumed to be smooth muscle cells, and surrounding negative cells presumed to be myofibroblastic in nature (g). A smoothelin immunostain (h) was diffusely negative in the lamina propria and only highlighted the smooth muscle fibers of the muscularis propria (bottom left corner).

the detrusor muscle/muscularis propria (pT2), or extend into the perivesical soft tissues (pT3). ${ }^{23}$ Although this might appear relatively straightforward, there are several well-known pitfalls in the pathological staging of bladder carcinoma ${ }^{5,10-12,22,24}$ that one should be aware of to avoid both understaging and overstaging of bladder carcinoma, both reported in a significant proportion of cases. ${ }^{3-5}$

One such caveat is misdiagnosing the smooth muscle of the muscularis mucosae as smooth muscle cells of muscularis propria suggesting a diagnosis of pT2 disease and leading to radical surgical management (cystectomy) for a tumor that would otherwise be treated conservatively (intravesical BCG therapy). This is a well-recognized problem especially in the evaluation of bladder biopsies and transurethral resection specimens..$^{7,8,10,11}$ A less recognized problem that we have occasionally encountered is cases in which myofibroblasts comprising the desmoplastic (or more cellular) spindle-cell response associated with invasive carcinoma (that is otherwise limited to the LP) had been misinterpreted as smooth muscle cells of the muscularis propria leading to a diagnosis of muscularis propria-invasive tumor. After encountering one such case in which the diagnosis was 'supported' by a positive smooth muscle actin immunostain in the spindle cells in question, we wanted to further characterize the immunohistochemical profile of these myofibroblasts as well as those of the smooth muscle cells of muscularis mucosae and smooth muscle cells of the muscularis propria to investigate the potential role of immunohistochemistry in distinguishing these cell types.

Accordingly, we evaluated the expression of different immunohistochemical markers that are known to be expressed in mesenchymal cells, contractile cells, or reactive stromal cells. We found that the different contractile markers were all very sensitive for detecting smooth muscle cells; however, in contrast to the other markers, both smooth muscle actin and actin were not sufficiently specific 

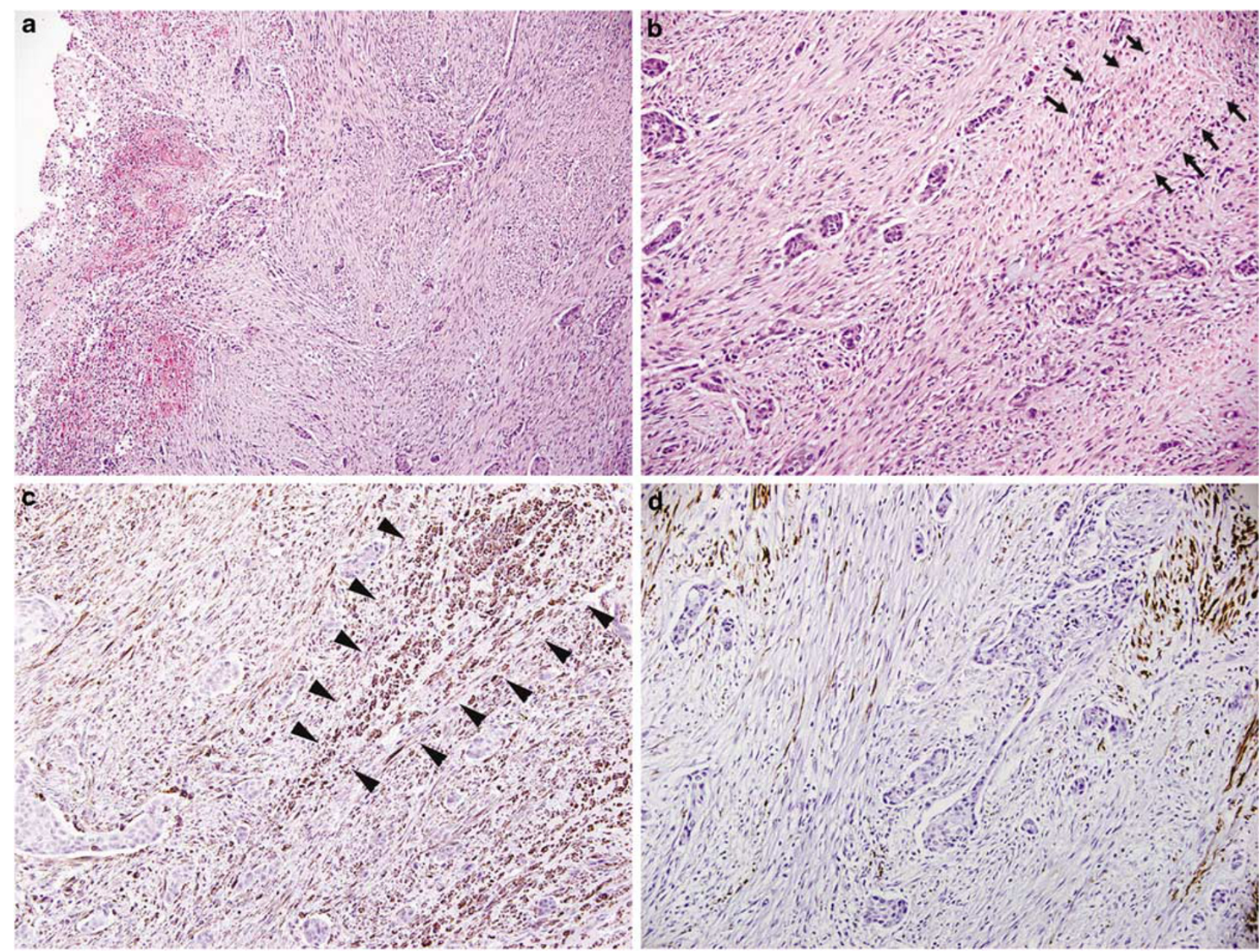

Figure 5 The utility of different immunostains in distinguishing the smooth muscle of the muscularis propria from a pseudosarcomatous reactive myofibroblastic response resembling smooth muscle fibers. A case of high-grade urothelial carcinoma associated with a cellular spindle-cell pseudosarcomatous response that is infiltrating between the fibers of the muscularis propria (a, b). The residual muscle fibers display more eosinophilic cytoplasm, most evident in the upper right hand corner of b (arrows). Compared to myofibroblasts within the cellular response, smooth muscle cells showed more intense staining for smooth muscle actin (c; arrowheads); however, desmin had better discriminatory power, as only smooth muscle cells were positive with this immunostain (d). Caldesmon and smoothelin immunostains (not shown) resulted in a pattern of staining similar to that of desmin (albeit with weaker intensity).

Table 4 The sensitivity, specificity, and accuracy rates of smoothelin and vimentin in distinguishing the smooth muscle cells of the bladder muscularis propria from the those of the muscularis mucosae

\begin{tabular}{|c|c|c|c|c|}
\hline Marker & Result cutoff & $\begin{array}{l}\text { Sensitivity (\%) } \\
\quad(95 \% \text { CI) }\end{array}$ & $\begin{array}{l}\text { Specificity (\%) } \\
(95 \% \text { CI) }\end{array}$ & $\begin{array}{c}\text { Accuracy }(\%) \\
(95 \% \text { CI })\end{array}$ \\
\hline Smoothelin & Positive (any intensity) & $100(75-100)$ & $36(12-68)$ & $73(52-88)$ \\
\hline Smoothelin & Positive $(\geq 2+)$ & $100(75-100)$ & $91(57-100)$ & $96(78-100)$ \\
\hline Smoothelin & Positive $(3+)$ & $100(75-100)$ & $100(68-100)$ & $100(84-100)$ \\
\hline Vimentin & Negative or $1+$ & $100(75-100)$ & $9(0-43)$ & $62(41-79)$ \\
\hline Vimentin & Negative & $93(66-100)$ & $82(48-97)$ & $76(59-87)$ \\
\hline Smoothelin and vimentin & Positive smoothelin $(\geq 2+)$ and negative vimentin & $93(66-100)$ & $100(68-100)$ & $96(78-100)$ \\
\hline Smoothelin and vimentin & Positive smoothelin $(\geq 2+)$ and negative or $1+$ vimentin & $100(75-100)$ & $100(68-100)$ & $100(84-100)$ \\
\hline
\end{tabular}

CI, confidence interval.

in distinguishing these cells from myofibroblasts. This is not surprising as myofibroblasts have previously been shown to express such 'smooth muscle-specific' markers. ${ }^{25-27}$ On the basis of this, we do not recommend using either of these two markers to evaluate histologically equivocal muscleinvasive bladder carcinoma. On the other hand, this study demonstrated that desmin, caldesmon, and 
smoothelin were all very specific for smooth muscle cells and may potentially complement hematoxylin and eosin-stained sections in this diagnostic setting. This high specificity and lack of desmin and caldesmon expression in myofibroblasts are consistent with earlier reports that demonstrated that bladder myofibroblasts ${ }^{19}$ or those associated with acute inflammation and tumor desmoplasia ${ }^{28}$ lack such expression; indeed recent reviews on the subject have emphasized that lack of desmin and caldesmon expression should be considered one of the characteristic features of myofibroblasts. ${ }^{27,29}$ It is unclear why this contrasts with myofibroblastic tumors of the bladder that are well known to express desmin and caldesmon in a significant proportion of cases. ${ }^{13}$

Because of the high sensitivity and specificity of desmin, caldesmon, and smoothelin immunostains for detecting smooth muscle cells, the addition of less discriminatory markers, such as vimentin and CD10, was not further useful for distinguishing them from myofibroblasts. Moreover, the wide variation in the intensity of CD10 staining of myofibroblasts as well as the lack of a valid internal control further curtailed its potential diagnostic utility.

On the other hand, we found absence of vimentin expression to be potentially useful in distinguishing the muscularis propria from the muscularis mucosae, as smooth muscle cells of the muscularis propria lacked such expression in all but one case, which contrasted with the smooth muscle cells of the muscularis mucosae in which most cases displayed weak or moderate expression. Given its ubiquitous nature in different cells of mesenchymal origin, lack of vimentin expression in smooth muscle cells of the muscularis propria was totally unexpected and a finding that we originally considered dismissing. A subsequent search of the literature, however, did reveal a recent study in which the smooth muscle cells of the muscularis propria from all eight cystectomy specimens evaluated by immunofluorescence were also found to be negative for vimentin. ${ }^{19}$ Moreover, the authors found that 'vimentin-positive cells were present between smooth muscle bundles, as well as in the microseptae surrounding their component fascicles, but not between individual smooth muscle cells', ${ }^{19}$ which is very similar to the moderate to strong immunohistochemical expression of vimentin we found in the endomysial and endothelial cells interspersed between the smooth muscle fibers of our cases. These findings appear to corroborate ours in that there is indeed a difference in vimentin expression levels between the smooth muscle cells of the muscularis mucosae and those of the muscularis propria.

Another marker we found useful in distinguishing muscularis propria from muscularis mucosae was smoothelin, as moderate to strong expression was $91 \%$ specific for smooth muscle cells of the muscularis propria, whereas strong expression was
$100 \%$ specific for muscularis propria. The expression of smoothelin in the bladder was first detected by Maake et $a l^{20}$ who found that the gene and protein (detected by RT-PCR and immunofluorescence, respectively) were expressed by the smooth muscle cells of the muscularis propria in 8 normal and 13 overactive bladders. Subsequently, Kuijpers et $a l^{19}$ found that protein expression was limited to the muscularis propria and was not identified in the muscularis mucosae in eight cystectomy specimens. This was most recently confirmed in a larger study by Lapetino et $a l^{21}$ who found that the smooth muscle cells of all 32 evaluated sections of muscularis propria displayed moderate or strong smoothelin expression in contrast to the smooth muscle cells of muscularis mucosae where moderate expression was only seen in 2 of 25 examples of hyperplastic muscularis mucosae and not in 32 examples of conventional muscularis mucosae. These findings are remarkably similar to ours despite probable variations in antibody dilutions and methodologies among the different studies.

As often is the case when different diagnostic tests are used in combination, we found that using vimentin and smoothelin in combination produced better sensitivity, specificity, and accuracy rates than when each immunostain was used individually. One option would be to use them in combination, as part of an algorithmic approach in the evaluation of foci of bladder carcinoma suspicious for muscularis propria invasion (Figure 6).

The fact that smoothelin has been shown to be expressed at later stages of smooth muscle differentiation $^{30,31}$ and/or in smooth muscle cells of the so-called contractile phenotype (rather than in those of the proliferative/synthetic phenotype) ${ }^{18,32}$ may account for the differences in the expression of this marker between the muscularis propria and muscularis mucosae. It can also be argued that similar developmental and functional considerations may explain the differential vimentin expression patterns of the smooth muscle in these two compartments. Obviously, more work is needed to investigate this.

Although the findings in this study clearly suggest that there is a potential role for immunohistochemistry in staging of bladder carcinoma, they are somewhat impacted by relying, to a certain extent, on different staining intensities in distinguishing between the muscularis propria and muscularis mucosae, which might create issues with reproducibility. Using vascular smooth as an internal reference for comparison would help in minimizing potential variations in intensity among different laboratories due to different fixation methods, antibody dilutions, etc. Although inclusion of a relatively small number of cystectomy specimens might also be considered another limitation, this was, by design, a preliminary exploratory study of different immunohistochemical markers in nonequivocal cases (where there was no question regarding the 


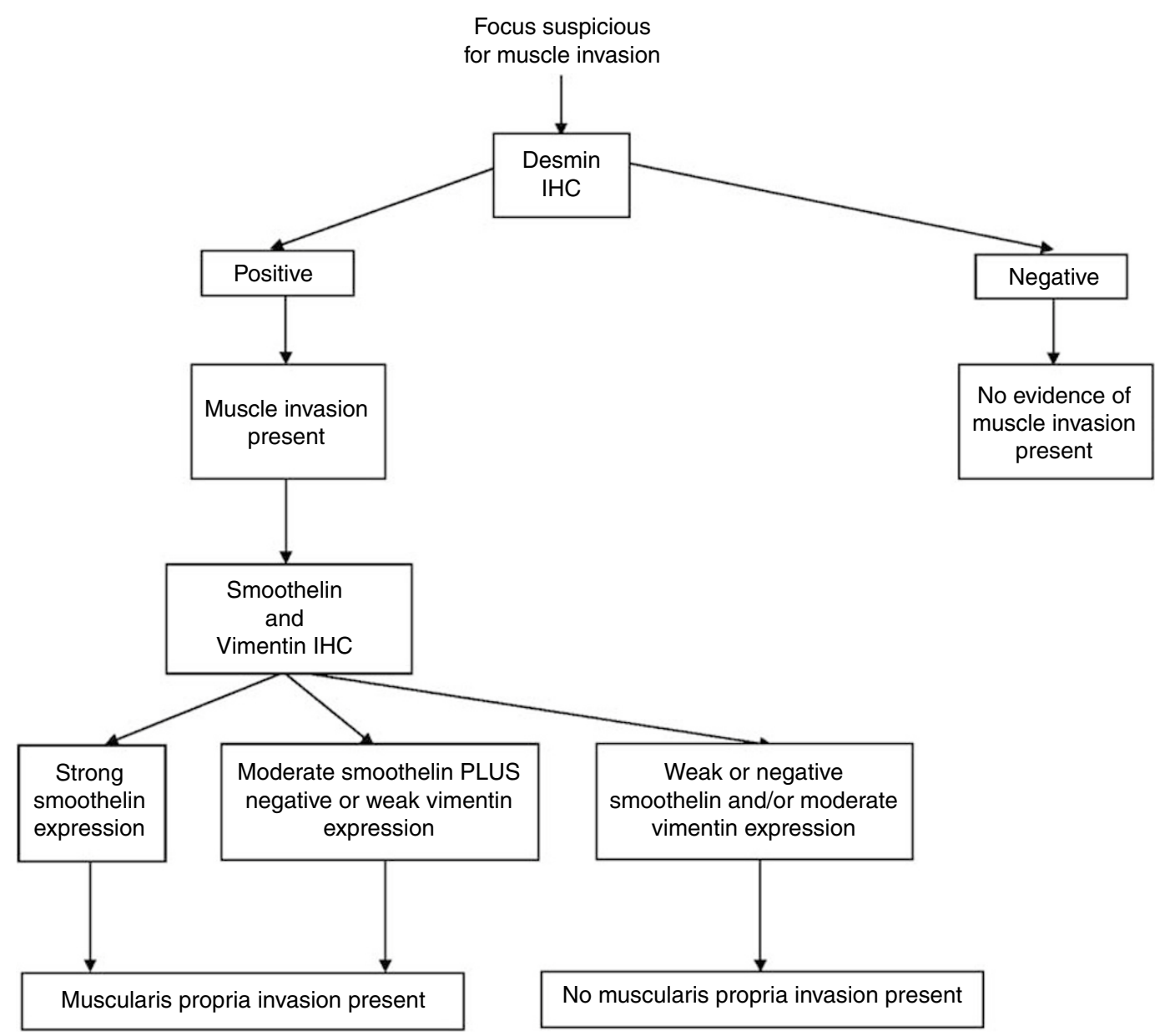

Figure 6 An algorithmic approach to the use of desmin, smoothelin, and vimentin immunohistochemistry in the evaluation of foci of bladder carcinoma suspicious for muscularis propria invasion.

depth of tumor invasion) to identify those immunostains that might be useful as an aid in staging of bladder carcinoma.

Our findings suggest that a panel composed of desmin, smoothelin, and vimentin immunostains, possibly using an algorithmic approach such as the one displayed in Figure 6, appears to have the most potential in this regard. Nevertheless, these results need to be validated in a larger sample of transurethral resection specimens (with questionable depth of invasion and where cystectomy follow-up staging data can be used as a gold standard) before more definitive conclusions can be made.

In summary, we have shown that differential expression of immunohistochemical markers can potentially distinguish between myofibroblasts and smooth muscle cells of the bladder, and also between the smooth muscle cells of the muscularis mucosae and those of the muscularis propria. Evaluation of additional cases, especially transurethral resection specimens that, as discussed above, are often the most difficult to stage, is warranted to further assess the potential role of immunohistochemistry in staging of bladder carcinoma. Finally, and as in any other diagnostic setting, we would caution that any number of immunohistochemically stained sections can only supplement and never replace morphological findings based on hematoxylin and eosin-stained sections.

\section{Acknowledgements}

This work was supported in part by a research grant from the Robert B and Jean G Adams Foundation to Dr Leona Council.

\section{References}

1 Jemal A, Siegel R, Ward E, et al. Cancer statistics, 2008. CA Cancer J Clin 2008;58:71-96.

2 Eble JN, Sauter G, Epstein JI, et al. Pathology and Genetics of Tumours of the Urinary System and Male Genital Organs. IARC Press: Lyon, France, 2004, 359p.

3 Cheng L, Neumann RM, Weaver AL, et al. Grading and staging of bladder carcinoma in transurethral resection 
specimens. Correlation with 105 matched cystectomy specimens. Am J Clin Pathol 2000;113:275-279.

4 Chang BS, Kim HL, Yang XJ, et al. Correlation between biopsy and radical cystectomy in assessing grade and depth of invasion in bladder urothelial carcinoma. Urology 2001;57:1063-1066; discussion 1066-1067.

5 Lopez-Beltran A, Cheng L. Stage pT1 bladder carcinoma: diagnostic criteria, pitfalls and prognostic significance. Pathology 2003;35:484-491.

6 Ro JY, Ayala AG, el-Naggar A. Muscularis mucosa of urinary bladder. Importance for staging and treatment. Am J Surg Pathol 1987;11:668-673.

7 Paner GP, Ro JY, Wojcik EM, et al. Further characterization of the muscle layers and lamina propria of the urinary bladder by systematic histologic mapping: implications for pathologic staging of invasive urothelial carcinoma. Am J Surg Pathol 2007;31:1420-1429.

8 Vakar-Lopez F, Shen SS, Zhang S, et al. Muscularis mucosae of the urinary bladder revisited with emphasis on its hyperplastic patterns: a study of a large series of cystectomy specimens. Ann Diagn Pathol 2007; 11:395-401.

9 Aydin A, Ucak R, Karakok M, et al. Vascular plexus is a differentation criterion for muscularis mucosa from muscularis propria in small biopsies and transurethral resection materials of urinary bladder? Int Urol Nephrol 2002;34:315-319.

10 Humphrey PA. Urinary bladder pathology 2004: an update. Ann Diagn Pathol 2004;8:380-389.

11 Epstein JI, Amin MB, Reuter VE. Bladder Biopsy Interpretation. Lippincott Williams \& Wilkins: Philadelphia, 2004, viii, 263p.

12 Young RH, Wick MR. Transitional cell carcinoma of the urinary bladder with pseudosarcomatous stroma. Am J Clin Pathol 1988;90:216-219.

13 McKenney JK, Amin MB. The role of immunohistochemistry in the diagnosis of urinary bladder neoplasms. Semin Diagn Pathol 2005;22:69-87.

14 Abdou AG. CD10 expression in tumour and stromal cells of bladder carcinoma: an association with bilharziasis. APMIS 2007;115:1206-1218.

15 Bircan S, Candir O, Kapucuoglu N, et al. CD10 expression in urothelial bladder carcinomas: a pilot study. Urol Int 2006;77:107-113.

16 Iwaya K, Ogawa H, Izumi M, et al. Stromal expression of CD10 in invasive breast carcinoma: a new predictor of clinical outcome. Virchows Arch 2002;440:589-593.

17 Ogawa H, Iwaya K, Izumi M, et al. Expression of CD10 by stromal cells during colorectal tumor development. Hum Pathol 2002;33:806-811.

18 van der Loop FT, Schaart G, Timmer ED, et al. Smoothelin, a novel cytoskeletal protein specific for smooth muscle cells. J Cell Biol 1996;134:401-411.
19 Kuijpers KA, Heesakkers JP, Jansen CF, et al. Cadherin11 is expressed in detrusor smooth muscle cells and myofibroblasts of normal human bladder. Eur Urol 2007;52:1213-1221.

20 Maake C, Landman M, Wang X, et al. Expression of smoothelin in the normal and the overactive human bladder. J Urol 2006;175:1152-1157.

21 Lapetino S, Paner GP, Venkataraman G, et al. Diagnostic utility of antibody to smoothelin in the distinction of muscularis mucosa from muscularis propria of the urinary bladder (abstract). Mod Pathol 2008; $21: 165 \mathrm{~A}$

22 Amin MB, Srigley JR, Grignon DJ, et al. Updated protocol for the examination of specimens from patients with carcinoma of the urinary bladder, ureter, and renal pelvis. Arch Pathol Lab Med 2003;127: 1263-1279.

23 Greene FL, American Joint Committee on Cancer, American Cancer Society. AJCC Cancer Staging Manual, 6th edn. Springer: New York, 2002, xiv, 421p.

24 Philip AT, Amin MB, Tamboli P, et al. Intravesical adipose tissue: a quantitative study of its presence and location with implications for therapy and prognosis. Am J Surg Pathol 2000;24:1286-1290.

25 Skalli O, Schurch W, Seemayer T, et al. Myofibroblasts from diverse pathologic settings are heterogeneous in their content of actin isoforms and intermediate filament proteins. Lab Invest 1989;60:275-285.

26 Jones H, Steart PV, Du Boulay CE, et al. Alpha-smooth muscle actin as a marker for soft tissue tumours: a comparison with desmin. J Pathol 1990;162:29-33.

27 Eyden B. The myofibroblast: phenotypic characterization as a prerequisite to understanding its functions in translational medicine. J Cell Mol Med 2008;12:22-37.

28 Ceballos KM, Nielsen GP, Selig MK, et al. Is antih-caldesmon useful for distinguishing smooth muscle and myofibroblastic tumors? An immunohistochemical study. Am J Clin Pathol 2000;114:746-753.

29 Eyden B, Banerjee SS, Shenjere P, et al. The myofibroblast and its tumours: a review. J Clin Pathol; published online: 17 October 2008 [e-pub ahead of print].

30 Ratajska A, Zarska M, Quensel C, et al. Differentiation of the smooth muscle cell phenotypes during embryonic development of coronary vessels in the rat. Histochem Cell Biol 2001;116:79-87.

31 Johansson B, Eriksson A, Ramaekers F, et al. Smoothelin in adult and developing human arteries and myocardium. Histochem Cell Biol 1999;112:291-299.

32 van der Loop FT, Gabbiani G, Kohnen G, et al. Differentiation of smooth muscle cells in human blood vessels as defined by smoothelin, a novel marker for the contractile phenotype. Arterioscler Thromb Vasc Biol 1997;17:665-671. 